\title{
Myotonia congenita in northern Finland: an epidemiological and genetic study
}

\author{
P Baumann, V V Myllylä, J Leisti
}

\begin{abstract}
An epidemiological and genetic investigation of myotonia congenita was carried out in northern Finland. Altogether 58 patients were identified (of whom 54 lived in the study area) in $\mathbf{2 3}$ families, with a prevalence of 7.3 per 100000 . The majority of the families originated from a sparsely populated area in western Lapland.

The mean age at onset of the disease was 11 years with a range of 2 to 45 years. The mean time that had passed before verification of the clinical disease was 18 (SD 14) years. The sex ratio $M / F$ was $2.2 / 1.0$.

Forty-seven cases were familial and 11 were sporadic. In six families/pedigrees the inheritance was compatible with autosomal recessive and in two families with autosomal dominant inheritance. In five additional families, in which autosomal recessive inheritance seemed most plausible, vertical transmission was also noticed. This could be explained either by consanguinity of the parents or by variant expression of the mutation(s) involved.

Our results suggest that myotonia congenita is unusually frequent in northern Finland, most probably as a consequence of an enrichment of the gene mutation(s) in the population.

$(\mathcal{H}$ Med Genet 1998;35:293-296)
\end{abstract}

Keywords: myotonia congenita; epidemiology; genetic aspects; Finland

Myotonia congenita (MC) is a non-dystrophic hereditary disease of skeletal muscle. MC can be inherited as an autosomal recessive trait (Becker's disease), as first described by Leyden, ${ }^{1}$ or as an autosomal dominant trait (Thomsen's disease) ${ }^{2}$ In both forms of MC, the characteristic clinical feature is myotonia, starting in early childhood. Myotonia is based on an electrical instability of the muscle membrane, suggested to be a direct consequence of a reduced chloride ion conductance in the tubular system of muscle fibres. ${ }^{3}$ During the first years the myotonic stiffness progresses slowly, but eventually affects the whole skeletal musculature. ${ }^{4}$ Myotonia is most severe during voluntary muscle activity following a period of rest. Another typical feature of the disease is variable muscle hypertrophy. Studies on MC patients ${ }^{5}$ have shown that the overall output of motor performance in the patients does not seem to be greatly impaired.

Recently, both the autosomal dominant and the autosomal recessive forms of the disease have been associated with mutations in the muscle chloride channel protein gene in human chromosome region $7 \mathrm{q} 35 .^{3}$ The mode of inheritance has also been questioned in some families, as the same mutation has been shown to lead to congenital myotonia in both heterozygous and homozygous forms. ${ }^{3}$

In previous epidemiological studies, ${ }^{47}$ the prevalence of the disease has been reported to range from 0.2 to 0.9 per 100000 . In Germany, the prevalence of autosomal recessive myotonia was reported as 0.3 per 100000 and of autosomal dominant myotonia 0.2 per 100000 , respectively. ${ }^{4}$

This study aimed to clarify the epidemiology and genetics of MC in northern Finland. The clinical findings will be reported separately.

\section{Patients and methods}

The study area consisted of the provinces of Oulu and Lapland and the northern part of the province of Vaasa, with a total population of 732000 . The basic family material consisted of the families seen at the Departments of Neurology and Clinical Genetics at the Oulu University Hospital. Additional patients were ascertained retrospectively from hospital records and registers of Oulu University Hospital and the central hospitals of Kajaani, Kokkola, Kemi, and Rovaniemi, from the years 1974 to 1995 . Furthermore, a call for patients with myotonic symptoms was addressed to the neurologists, paediatric neurologists, and general practitioners in the area of the investigation. The obtainable first degree relatives (healthy sibs included) were studied for microsymptoms, both clinically and electrophysiologically, in order to exclude or verify signs of myotonia.

The clinical diagnostic and inclusion criteria for the disease were as follows: muscle stiffness, muscle function improving with continuing exercise (action myotonia), variable muscle hypertrophy, and myotonic bursts on intramuscular electromyography (EMG). The clinical criteria are in accordance with those set out by the European Neuromuscular Centre Workshop. ${ }^{8}$

All the patients underwent a careful neurological and EMG examination. Genetic studies included family history and, in selected cases, tracing of common ancestors with the help of church records.

For the prevalence estimate, all patients living in the study area in 1995 were included. The evaluation of onset of the disease was based on the history given by the patients or their parents or both.

The study protocol was approved by the Ethics Committee of the Medical Faculty, 


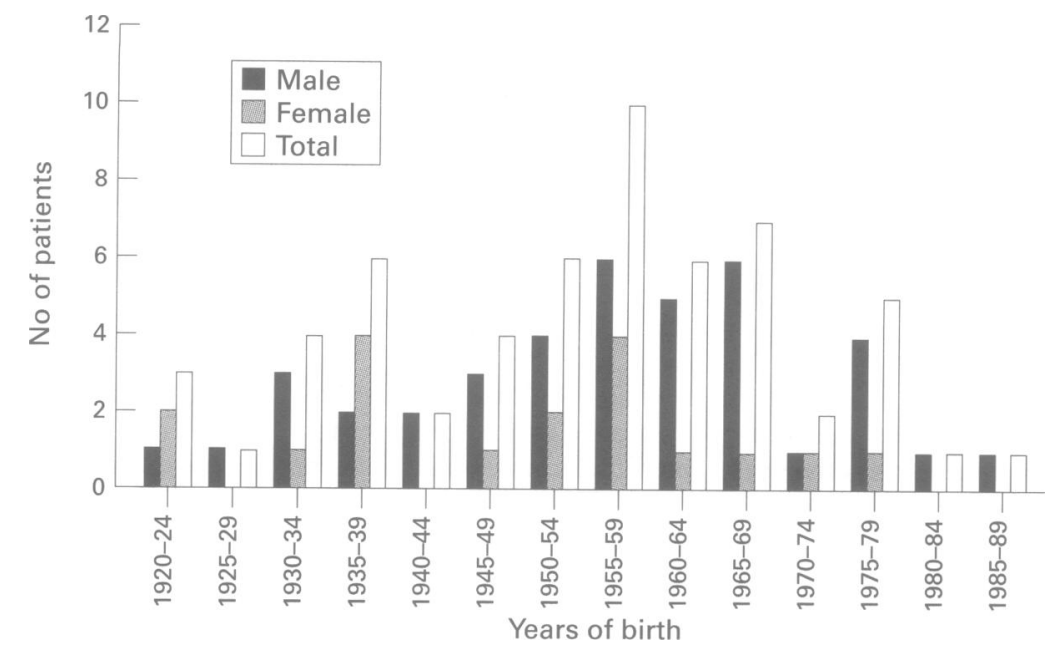

Figure 1 Sex specific distribution of myotonia congenita patients in northern Finland according to the year of birth between 1920-1989.

University of Oulu. Informed consent was obtained from each subject participating in the study.

\section{Results}

The main results are given in figs 1,2 , and 3 . Fifty-four patients (39 males and 15 females) with $M C$ were detected, giving a prevalence of 7.3 per 100000 for the area investigated. The mean age of the patients was 41 years (range 6 to 75 ). From the years 1950 to 1979 it could be roughly estimated that 1.2 patients were born annually in the area investigated (fig 1). The domiciles of the patients as shown in fig 2 show a clustering of the disease for the cases in western Lapland.

The mean age at onset of symptoms was reported as 11 years and the majority (37 patients, 65\%) fell in the age group between 6 and 10 years. Two of the patients experienced motor disturbance first after 16 years of age and one patient before 6 years of age. Two patients were not aware of myotonia before participating in the study. The mean time that elapsed before the diagnosis of the disease was 18 (SD 14, range 1 to 43) years.

The patients belonged to 23 families. Fortyseven cases were familial and 11 were sporadic (fig 3). Twelve patients belonged to a large family (A), which could be traced back to the late 18th century, originating from western Lapland. In this family several instances of either parental consanguinity or close relationship with parents of other patients were found. In three additional families (B, C, and F, 16 patients) with probable autosomal recessive inheritance of the disease, vertical transmission was also seen. In two families (D and E, six patients) transmission was compatible with an autosomal dominant mode of inheritance. In six families ( $G$ to $L, 13$ patients) autosomal recessive inheritance was most plausible. The rest of the cases ( $M$ to $W, 11$ patients) were sporadic.

\section{Discussion}

The prevalence of $\mathrm{MC}$ in northern Finland, 7.3 per 100000 , is exceptionally high and partly reflects the systematic search for patients

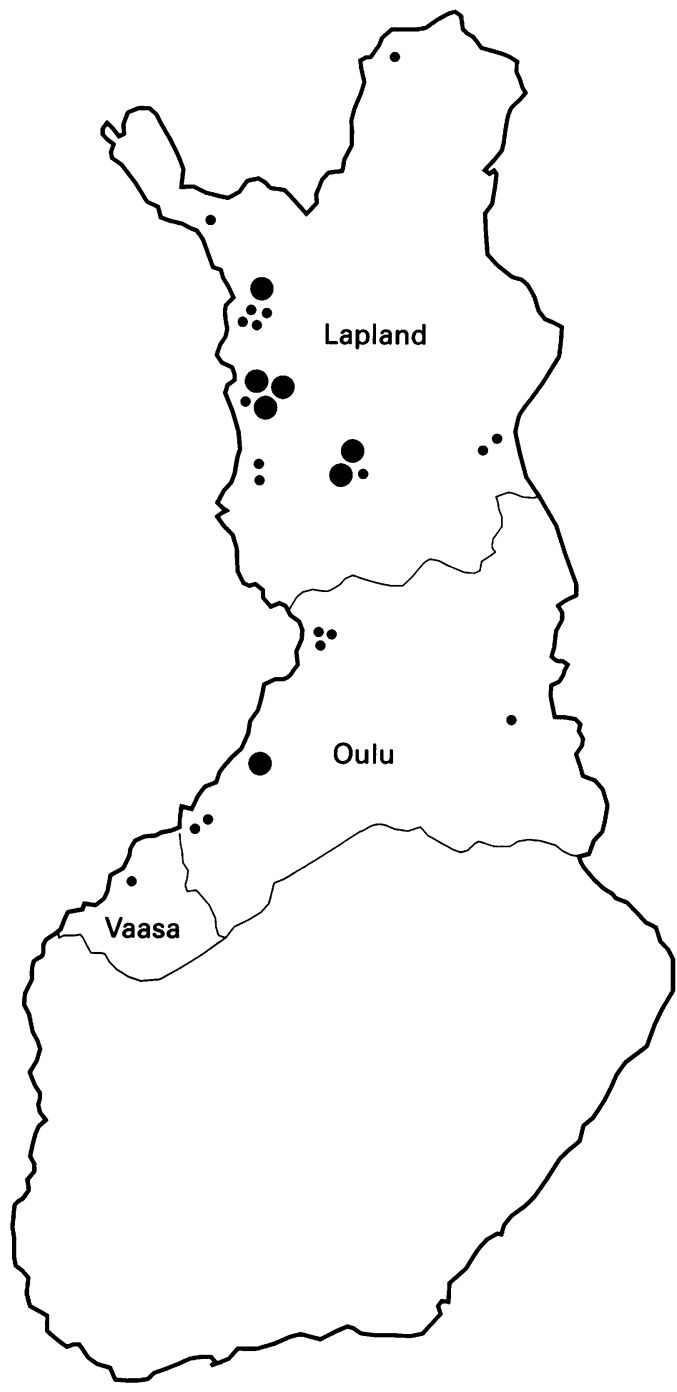

Figure 2 The map of Finland. The areas of investigation are marked with thin lines and the domiciles of the patients with myotonia congenita with filled circles. Large circles mark five patients and small circles mark one patient.

both from medical records and from close family members. On the other hand, the high prevalence rate could also be explained by a founder effect or careful neurological and EMG examination; thus even relatively mildly affected people could be included. As shown in fig 1 , it can be roughly estimated that 1.2 cases are born annually. It is also probable that the disease is more common in the area studied for genetic reasons. Several rare recessive diseases have accumulated in the Finnish population owing to the enrichment of the respective gene mutations. $^{910}$ It is possible that the gene(s) causing MC have also been enriched, especially in western Lapland, by the same mechanism of isolation by distance as in the other diseases of the Finnish disease heritage. There is only one larger study on the frequency of MC. ${ }^{4}$ In this study from West Germany and West Berlin, a frequency of 0.2 per 100000 (116 cases) for dominantly inherited and 0.3 per 100000 (150 cases) for recessively inherited clinically verified myotonia were reported, respectively. Incomplete ascertainment of the patients was, however, assumed. In a smaller study by Pinessi et al (10 cases), a prevalence rate of 0.9 
per 100000 from the city of Turin, Italy, was reported.

In our study the sex ratio was 2.2:1.0 in favour of males. In the German study of Becker, ${ }^{4}$ it was assumed that, in the dominant form of the disease, the sex ratio is approximately even and in the recessive form a slight preponderance of male patients was found. Our results indicate that the disease is clearly more common in men than in women. The reason for this is unknown.
The mean age at onset of myotonia as reported by our patients or their parents was 11 years; $65 \%$ of the patients experienced myotonia between their 6th and 10th year of life and an additional $27 \%$ before the age of 16 . The true onset of the disease process was probably earlier, as shown by the two asymptomatic cases who were detected by the EMG registration. It is also possible that the early symptoms of the disease, that is, mild motor performance disturbance, have not been recog-

A

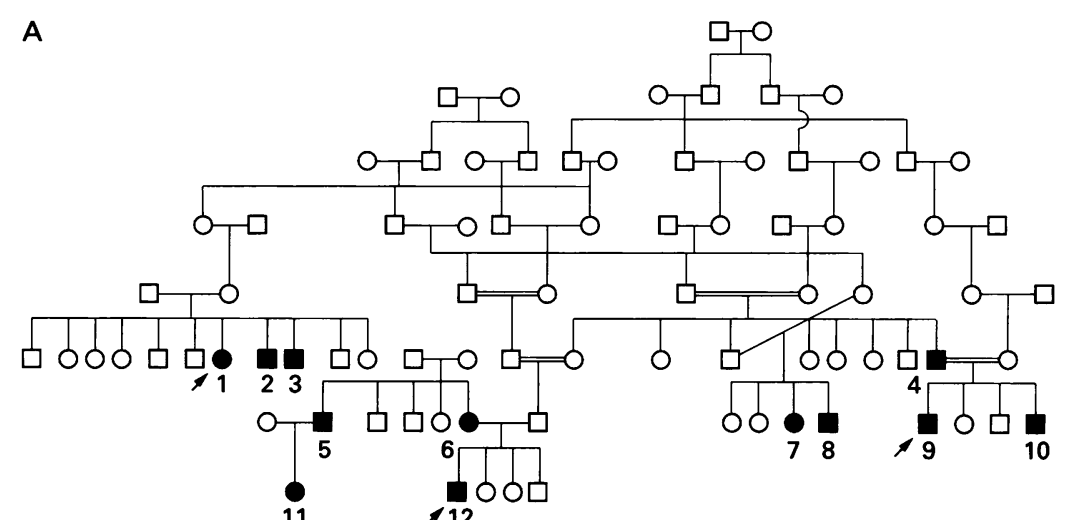

B
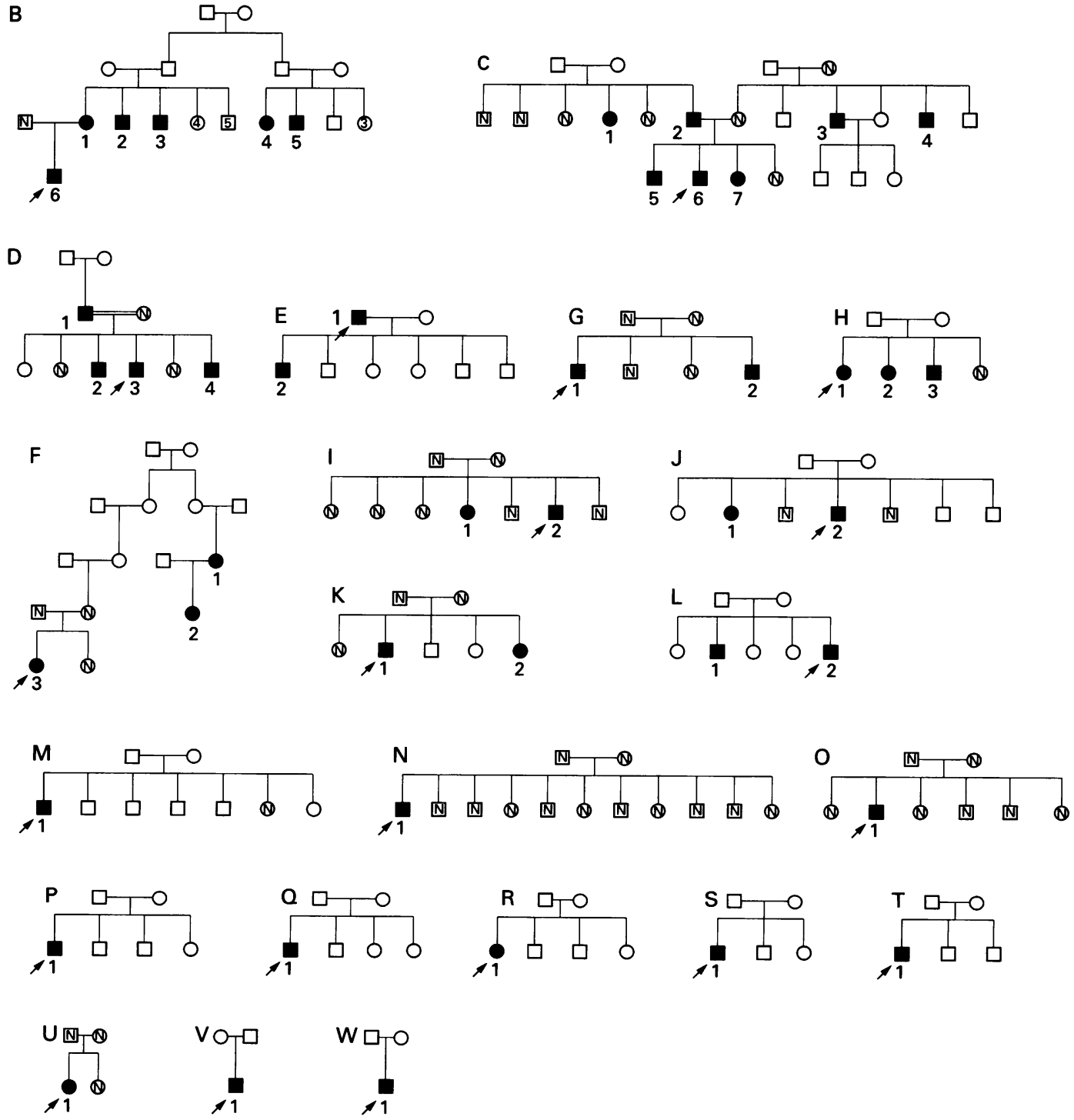

Figure 3 Pedigrees of families $A$ to W. Probands are indicated by an arrow, affected cases are fully shaded. $N$ indicates persons examined and found to be normal and double lines parental consanguinity. 
nised as symptoms of the disease. This could also explain the relatively long time that was needed for diagnosis (18 (SD 14) years). Becker $^{4}$ suggested that dominant myotonia most often starts in the first three years of life, whereas the usual age at onset in recessive myotonia is between the fourth and twelfth years. The disease is thus often experienced as relatively mild and medical aid is sought late. This assumption is supported by the findings of Baumann et $a l,{ }^{56}$ who suggested that the overall output of the motor performance of myotonic patients is not greatly impaired. On the other hand, MC patients keep their myotonia a secret, even from general physicians. ${ }^{4}$ The rapid development of neurological diagnostics during the last decades has shortened the delay in reaching a diagnosis, as seen also in the present material.

Even when vertical transmission of the disease was seen in several families, autosomal recessive inheritance seems the most plausible mechanism to explain most of the familial occurrence of the disease. In the largest pedigree (A), several instances of parental consanguinity could be shown to explain the occurrence of the disease, both in parents and their children. It is also possible that a mutation(s) prevails in the population which allows the disease to become manifest in heterozygous and homozygous forms. ${ }^{10} \mathrm{Mo}-$ lecular studies that are in progress should explain the genetic features of this exceptional prevalence of the disease in northern Finland.

We conclude that $\mathrm{MC}$ is unusually frequent in northern Finland, most probably as a consequence of enrichment of the gene(s) in the population.

1 Leyden E. Klinik der Rückenmarkskrankheiten. Vol 1. Berlin: Hirschwald, 1874

2 Thomsen J. Tonische Krämpfe in willkürlich beweglichen Muskeln in Folge ererbter psychischer Disposition (Ataxia muscularis) Arch Psychiatr Nervenkrankh 1876;6:702-18.

3 Meyer-Kleine C, Steinmeyer K, Ricker K, Jentsch T, Koch $M$. Spectrum of mutations in the major human skeletal muscle chloride channel gene (CLCN I) leading to myotonia. Am f Hum Genet 1995;57:1325-34.

4 Becker PE. Myotonia congenita and syndromes associated with myotonia. Stuttgart: Thieme, 1977.

5 Baumann P, Vanharanta H, Kauranen K, Myllylä V. Myotonia congenita: quantitation of different aspects of motor performance. Eur Neurol 1995;35:206-11.

6 Baumann P, Siira P, Vanharanta H, Myllylä VV. Quantification of muscle strength in recessive myotonia congenita. Eur Neurol 1996;36:284-7.

7 Pinessi L, Bergamini L, Cantello R, Di Tizio C. Myotonia congenita and myotonic dystrophy: descriptive epidemiological investigation in Turin, Italy (1955-1979). Ital $\mathcal{F}$ Neurol Sci 1982;3:207-10.

8 Workshop Report. Non-dystrophic myotonias and periodic paralyses. Neuromusc Disord 1993;3:161-8.

9 de la Chapelle A. Disease gene mapping in isolated human populations: the example of Finland. $\mathcal{F}$ Med Genet 1993;30: 857-65.

10 Norio $R$, Nevanlinna HR, Perheentupa J. Hereditary diseases in Finland; rare flora in rare soil. Ann Clin Res 1973;5:109-41. 